\title{
Diabetes and Glycemic Control in Necrotizing Otitis Externa $(\mathrm{NOE})$
}

\author{
Chilaf Peled ${ }^{1}$, Re'em Sadeh² ${ }^{2}$ Sabri El-Saied ${ }^{1}$, Viktor Novack$^{2}$, and Daniel M. Kaplan ${ }^{1}$ \\ ${ }^{1}$ Soroka University Medical Center \\ ${ }^{2}$ Soroka Medical Center
}

July 20, 2020

\begin{abstract}
Introduction: Although the association between necrotizing otitis externa (NOE) and diabetes mellitus (DM) is well known, there is little knowledge in regards to the effects of DM and glycemic control (preadmission and during hospitalization) on the outcome of NOE. The aim of the study was to determine the effects of DM duration and preadmission glycemic control, and in-hospital glycemic control on NOE severity. Methods: A retrospective case series analysis, including all patients between the years 1990-2018 hospitalized due to NOE were included in the study. Data collected included NOE disease characteristics, duration of DM, DM associated comorbidities, glycated hemoglobin (HbA1c), urine micro-albumin and in-hospital blood glucose measurements. Disease severity was defined based on duration of hospitalization (above or below 20 days) and need for surgery. Results: Eighty nine patients were included in the study. Eighty three patients (94.3\%) had DM. Preadmission HbA1c was 8.13\% (5.8\%-12.6\%). Forty nine patients (65.5\%) had mean blood glucose of [?]140mg \dL and 26 patients (34.5\%) had [?] $140 \mathrm{mg} / \mathrm{dL}$. DM duration was 157.88 months among NOE patients who required surgery, and 127.6 months among patients who were treated conservatively (p-value 0.25 ). HbA1c in patients hospitalized $<20$ days was $7.6 \%$, and $8.7 \%$ among NOE hospitalized [?] 20 days (p-value 0.027). Seven patients with mean blood glucose of [?]140mg \dL had Pseudomonas Aeruginosa (PA-NOE) (26.7\%), in comparison to 25 patients (51.0\%) with mean blood glucose measurement of [?]140mg $\backslash \mathrm{dL}(\mathrm{p}=0.045)$. Conclusions: HbA1c levels at admission are associated with longer hospitalization duration among NOE patients. High mean blood glucose during hospitalization was associated with a higher likelihood for a PA infection, however it had no effect on disease outcome.
\end{abstract}

\section{Key points:}

- Although the association between necrotizing otitis externa (NOE) and diabetes mellitus (DM) is well known, there is little knowledge in regards to the effects of DM and glycemic control on the outcome of NOE.

- The aim of the study was to determine the effects of DM duration and preadmission glycemic control, and in-hospital glycemic control on NOE severity. Disease severity was defined based on duration of hospitalization (above or below 20 days) and need for surgery.

- Eighty nine patients were included in the study. DM duration was 157.88 months among NOE patients who required surgery, and 127.6 months among patients who were treated conservatively (p-value 0.25). HbA1c in patients hospitalized $<20$ days was $7.6 \%$, and $8.7 \%$ among NOE hospitalized [?] 20 days (p-value 0.027).

- Seven patients with mean blood glucose of [?]140mg \dL had Pseudomonas Aeruginosa-NOE (26.7\%), in comparison to 25 patients (51.0\%) with mean blood glucose measurement of [?]140mg $\backslash \mathrm{dL}(\mathrm{p}=0.045)$.

- HbA1c levels at admission are associated with longer hospitalization duration among NOE patients. High mean blood glucose during hospitalization was associated with a higher likelihood for a PA infection, however it had no effect on disease outcome. 
Key words: Malignant, Otitis externa, Diabetes Mellitus, Necrosis, Neuropathy.

\section{Introduction:}

Necrotizing otitis externa (NOE) is a severe inflammatory process affecting the external ear and skull base. This condition is commonly seen among elderly patients with diabetesmellitus(DM), with a reported prevalence of $90-100 \% \%^{1,2}$. The most common pathogen associated with NOE is Pseudomonas Aeruginosa (PA), however the incidence of fungal and sterile culture NOE is rising ${ }^{3,4}$. Classically, surgery was the most common treatment for NOE and mortality rateswere as high as $50 \%^{5}$. Nowadays, with the introduction of anti-PA antibiotics, long term antibiotic treatment is the main stay, with surgery preserved for selected cases.

Although the association between NOE and DMis well established, there is littleknowledge in regards to the effects of DM duration and glycemic control with disease progression and outcome.Joshua et al ${ }^{6}$ reported that a history of diabetic related complications was associated with longer treatment duration andshorter survival in NOE patients. In contrast,Lohet $\mathrm{al}^{7}$ reported that HbA1c levels, which reflect recent glycemic control, were not associated with disease outcome. Similarly, Franco-Vidal et al $^{8}$ reported that DM is not a prognostic factor in NOE.

In light of these controversies and limited data the aims of our study were:1. Examine the effect DM duration, as well aspreadmission glycemic control with NOE outcome. 2. Examine the effect of glycemic control during hospitalization on NOE outcome. 3. Observe trends in the incidence of the offending pathogens in regards to DM severity and glycemic control.

\section{Methods and materials:}

A retrospective case series analysis was conducted, including all patients hospitalized due to NOE between the years 1990-2018. The research was conducted in compliance and approval of the requirements of the medical center's institutional review board; the Human Subject Research Committee.

Inclusion criteria included: 1) patients 18 years and above 2) diagnosed NOE, based on the Cohen and Friedman classification ${ }^{9} 3$ ) absence of chronic middle ear disease 4) no history of ear surgery due to chronic ear disease 5) hospitalization duration of more than 7 days. Exclusion criteria included: 1) patients younger than 18 years, 2) history of chronic middle ear disease 3 ) patients who underwent surgery due to chronic ear disease 4) hospitalization duration of less than 7 days.

Data collected included: 1) patients age and gender 2) complaints at admission 3) physical findings at admission 4) laboratory findings 5) radiological imaging 6) culture results7) antibiotic treatment 8) type of surgery performed 9)duration of hospitalization.

Medical and laboratory data retrieved in order to evaluate DMincluded: 1) duration of DM 2) medical comorbidities associated with DM at time of admission 3) hemoglobin A1c levels (HbA1c) reflecting DM control at time of admission 4) Most recentmicro-albuminuria levels, defined as microalbuminuria collected up to six months prior to admission 5) Blood glucose measurements duringhospitalization. For statistical analysis, and based on the American diabetes association ${ }^{10}$, mean blood glucose measurements were divided into two groups using a cutoff of $140 \mathrm{mg} / \mathrm{dL}$.

Two main measures were used in order to evaluate disease outcome: 1) duration of hospitalization - patients hospitalized more or less than 20 days 2) need for surgical intervention under general anesthesia.

\section{Treatment protocol:}

Upon admission, all patients received empiric treatment comprising of quinolone or third generation cephalosporin. Further treatment was based on the pathogen's sensitivity profile. In cases where no pathogen was isolated, empiric intravenous anti-PA antibiotic was continued. Anti-fungal treatment was initiated only in cases of positive cultures. 
All patients underwent daily examination and local debridement. Granulation tissue was removed and sent for pathological evaluation in all patients. Indications for surgery were 1) no response to conservative treatment, as assessed by a senior otologist 2) new onset facial palsy or presence of facial nerve palsy not responding to treatment.

\section{Statistical analysis:}

For our population characterization, continuous variables were presented as mean \pm standard deviation (SD), and categorical variables were presented as frequency and percentages. In a univariable analysis we compared demographic, clinical and hospitalization characteristics between every two groups; surgical treatment (yes $\backslash$ no), admission duration (more and less than 20 days) and glucose levels (mean glucose [?] 140, mean glucose [?] 140). Parametric variables were compared using t-tests and categorical variables were compared with Chi-square or Fisher exact tests. Variables found to be nonparametric were compared using MannWhitney test.All p-values were based on a two-tailed test of significance, with a p-value $<0.05$ considered as significant. All statistical analyses were conducted using SPSS Version 25 (SPSS Inc., Chicago, IL)

\section{Results:}

Eighty-nine patients were included in the study, accounting for 91 affected ears. The study group included 53 males (59.5\%) and 36 females (40.5\%), with a mean age of 69.8 years. Eighty-three (94.3\%) patients had $\mathrm{DM}$ at time of admission. Table-1 depicts patient's characteristics.

The most common complaints at admission were otalgia $(n=79,86.8 \%)$ and aural discharge $(n=43,47.2 \%)$. Edema of the external ear canal $(\mathrm{n}=67,73.6 \%)$ was the most common finding on physical examination, followed by Granulation tissue $(n=66,72.5 \%)$ and aural discharge $(n=44,48.3 \%)$. Six patients $(6.5 \%)$ had facial palsy.

Swab cultures were taken from all patients at admission. PA-NOE was the most common pathogen $(\mathrm{n}=41$, $45.0 \%)$, followed by sterile-NOE $(\mathrm{n}=19,20.8 \%)$, and candida species $(\mathrm{n}=11,12.0 \%)$. For statistical analysis we excluded those with missing data, and grouped the results into 4 main categories:1) PA-NOE (n=41) 2)sterile-NOE (n=19) 3)Fungal-NOE (n=14) 4) other bacteria's $(\mathrm{n}=4)$.

Mean duration of DM at time of hospitalization was 136.6 months, ranging from 6-396 months. The most common DM associated complications were retinopathy $(\mathrm{n}=26,30.9 \%)$, nephropathy $(\mathrm{n}=24,28.5 \%)$ and peripheral neuropathy $(\mathrm{n}=16,19.0 \%)$. Mean HbA1c, which wasretrieved from 28 patients, was $8.13 \%(5.8 \%$ $12.6 \%$ ). Mean urine microalbumin, collected from 40 patients was $195.5 \mathrm{mcg}$. Blood glucose measurement during hospitalization was collected from 75 effected ears. Forty-nine patients $(53.8 \%)$ had mean blood glucose measurements of [?]140mg $\backslash \mathrm{dL}$ and 26 patients (28.5\%) had [?] 140mg/dL. Table-2 depicts DM related patient characteristics.

Average duration of hospitalization was 22.3 days. Twenty five patients (27.4\%) required surgical intervention. At time of data collection 55 patients died, among them 3 died during hospitalization.

Tables 3 and 4 evaluate the relationship between DM characteristics and NOE outcomes. Mean duration of diabetes was 157.88 months among NOE patients who required surgery in comparison to 127.6 months among patients who were treated conservatively (p-value 0.25$)$. Thirty six patients $(54.5 \%)$ had mean glucose levels [?]140 $\mathrm{mg} \backslash \mathrm{dLin}$ the non-surgical group, as opposed to 13 patients $(52.0 \%)$ in the surgical group (pvalue 0.69 ). No statistical difference was seen between the two groups in regards to HbA1c levels (p-value 0.59 ) and microalbuminuria (p-value 1.0).Fifty one patients were hospitalized less than 20 days (56.0\%) and 40 patients (44\%) were hospitalized longer than 20 days. Average age was 68.3 years among patients hospitalized less than 20 days in comparison to 71.7 years among people hospitalized more than 20 days (p-value 0.15). Mean HbA1camong NOE patients hospitalized less than 20 days was $7.6 \%$ in comparison to $8.7 \%$ among patients hospitalized [?]20 days (p-value 0.027). Duration of diabetes (p-value 0.65), long term complications of diabetes ( $\mathrm{p}$-value 0.45 ) and mean glucose measurements during hospitalization (p-value 0.16 ) were not statistically significant between the two groups. 
In order to further evaluate the effects of glycemic control during hospitalization on NOE, we compared patients with mean blood glucose measurements above and below $140 \mathrm{mg} \backslash \mathrm{dL}$ (Table-5), regardless of disease outcome. Among patients with blood glucose measurements of [?]140mg $\backslash \mathrm{dL}, 11$ patients had long term complications of DM (42.3\%) in comparison to 30 patients $(61.2 \%)$ with mean blood glucose measurements of[?]140mg $\backslash \mathrm{dL}(\mathrm{p}=0.11)$. Duration of DM was associated with higher mean glucose measurements during hospitalization $(\mathrm{p}=0.005)$. Similarly,higher levels of $\mathrm{HbA1C}$ were associated with higher mean glucose measurements during hospitalization $(\mathrm{p}=0.001)$. Sevenpatients with glucose measurements [?]140mg $\backslash \mathrm{dL}$ had PA-NOE (26.7\%), in comparison to 25 patients (51.0\%) among those with glucose measurements[?]140mg $\backslash \mathrm{dL}$ $(\mathrm{p}=0.045)$. Figure -1 presents the incidence of isolated pathogen based on mean glucose measurements during hospitalization.

\section{Discussion:}

The effect of DM on NOE is not clearly understood. One may intuitively speculate that glycemic control prior and during admission is of paramount importance in the treatment of NOE. However,is this in fact true? Is an uncontrolled patient more likely to be infected with a certain pathogen or is he at a greater risk for a worse outcome? Perhaps the main reason for not having a validated answer is the relative rarity of the disease and the small case series published from different centers, during the last few decades. Our group previously reported on 83 ears in 81 patients with $\mathrm{NOE}^{3}$. We found that duration of complaints prior to admission and the presence of aural discharge were associated with longer hospitalization length. Additionally, advanced age was associated with conservative treatment failure and the need for surgery. In this research, we have slightly enlarged the study group, and focused on the effects of DM and glycemic control on NOE progression, using the same end-point parameters- length of admission and the need for surgery.To the best of our knowledge this study represents the largest and most detailed research evaluating the relationship between DM and NOE.

The diagnosis of NOE is still not in complete agreement and there are more than 20classification systems ${ }^{4}$ reported in the literature. In our institute we diagnosed NOEbased on the Friedman and Cohen criteria $^{9}$, which uses obligatory and occasionalclinical parameters. Although not all patients exhibited all the required parameters at admission, repeated physical examination during hospitalization revealed all obligatory criteria's, confirming the diagnosis of NOE.

Similar tothe findings in previous studies, our patients with NOE were mostly elderly and $93.2 \%$ of themwere known diabetics. Also in agreement with the literature, otalgia and aural discharge were the most common complaints at admission, and edema of the external ear canal and granulation tissue were the most common clinical findings at admission.

PA is considered the most common pathogen in NOE. Despite that, there have been numerous publications indicating a shift in the incidence of the offending pathogen and an increase in the rate of fungal NOE and sterile $\mathrm{NOE}^{3,4}$. In the current study PA-NOE was the most common pathogen (45.0\%), followed by sterile culture (20.8\%) and fungal-NOE (15.3\%). When comparing these results with previously reported data,we have observed a continuing decline in PA-NOE and an increased in fungal-NOE ${ }^{3}$.

Our understanding in regards to the effects of DM on NOE progression is not clear, however several key elements in the pathophysiology and treatment of NOE share resemblance to diabetic foot ${ }^{11}$, which is caused due to DM related peripheral vascular pathology. Initially, both NOE and diabetic foot occur as a result of direct spread of soft tissue inflammation into the bone, causing local osteomyelitis. Second, in both entities PA is a leading pathogen in the inflammatory process. Last, treatment protocol is similar and comprised of long term antibiotic treatment and surgery in selected cases. These similarities support previous assumptions that microangiopathies are probably the main pathology leading to NOE among DM patients ${ }^{7,11}$. If this is the case, it is reasonable to assume that DM duration and severity effects NOE progression.

There are limited studied on the relationship between DM and NOE, using different parameters to assess both DM severity, and NOE progression. Joshua et $\mathrm{al}^{6}$ found that NOE patients that exhibited all obligatory parameters by the Friedman and Cohen criteria, had a higher incidence of DM, higher use of oral antidiabetic 
medications, and higher incidence of DM related complications in comparison to NOE patients that did not show all obligatory parameters. Duration of diabetes prior to admission, HbA1c levels and microalbuminuria however, were not evaluated. Stern-Shavitet $a 1^{12}$ reported on patients from the same center and found that DM correlated and predicted disease specific mortality, however DM duration and severity were not analyzed. Leeet al. ${ }^{13}$ reported that DM duration was associated with uncontrolled NOE, but HbA1c was not associated with NOE progression. Similarly, Lohet al. ${ }^{7}$ reported that DM severity, defined by HbA1c $>7.0 \%$ was not associated with the disease outcome. Our study focused on DM severity by analyzing three parameters DM duration, HbA1c levels and microalbuminuria, which is commonly used to assess renal dysfunction as a result of long lasting vascular pathology.

In contrast to previous studies our results found that $\mathrm{HbA1c}$ levels were associated with a longer hospitalization duration among NOE patients $(\mathrm{p}=.027)$. DM duration and microalbuminuriadid not correlate with need for surgery and duration of hospitalization. This might indicate that diabetic control at the time of disease onset plays a more substantial role in the insemination of bacteria into the surrounding bone, in comparison to overall DM duration, thus leading to a severe disease requiring longer in hospital treatment.

Apart from DM severity, the importance of glycemic control during hospitalization was also reported by several publications. Carfrae et al. ${ }^{14}$ reported that strict glycemic control is an essential principle in NOE treatment. Similarly, Hollies ${ }^{5}$ reported that tight glycemic control is one of the key management strategies for the treatment of NOE. In contrast, Chen et al. ${ }^{15}$ reported that average glycemic levels among NOE patients were not associated with mortality in NOE patients. Also, Lee et al. ${ }^{13}$ reported that mean glucose levels were not statistically significant between controlled and uncontrolled NOE. In the presented study, mean glycemic levels during hospitalization were associated with DM duration $(\mathrm{p}=.005)$ and HbA1C levels ( $\mathrm{p}$-value 0.001), but were not associated with hospitalization duration or need for surgery. This may show that strict glycemic control during hospitalization is not as important as previously mentioned in the treatment and outcome of NOE patients.

Interestingly, higher glycemic levels during hospitalization were associated with PA-NOE $(\mathrm{p}=.045)$. This might be explained by the fact that DM is known to cause a dysfunction in the phagocytotic activity of polymonuclear cells and macrophages leading to increased sensitivity of PA infection ${ }^{16}{ }^{17}$. The possible relevance of this finding might be important in sterile NOE. In such patients, poor glycemic control during hospitalization can support the continuing use of anti-PA antibiotics

\section{Limitations:}

We consider several limitations to our study:

1) The diagnosis of NOE is clinical, and represents an extension of severe otitis externa.Although unlikely,It is still possible that some of the patients had severe otitis externa rather than NOE.

2) The parameters of micrabuminemia and $\mathrm{HbA} 1 \mathrm{C}$ were only partially available (in the more recent cases). This missing data limits the possibilities of better statistical analysis and therefor limits conclusions on their effect on the outcome of NOE

\section{Conclusions:}

NOE is a severe life threatening condition, strongly associated with DM patients. Our findings demonstrate that HbA1c levels at admission are associated with increased hospitalization duration among NOE patients. Furthermore, glycemic control during hospitalization is associated with higher likelihood of PA infection but did not effect outcome. Duration of DM and microalbuminuria were not associated with hospitalization length and need for surgery.

\section{Conflict of interest:}

The authors declare no conflict of interest and no financial support

\section{References:}


1. Berenholz L, Katzenell U, Harell M. Evolving resistant pseudomonas to ciprofloxacin in malignant otitis externa. Laryngoscope . 2002;112(9):1619-1622. doi:10.1097/00005537-200209000-00017

2. Mani N, Sudhoff H, Rajagopal S, Moffat D, Axon PR. Cranial nerve involvement in malignant external otitis: implications for clinical outcome. Laryngoscope . 2007;117(5):907-910. doi:10.1097/MLG.0b013e318039b30f

3. Peled C, El-Seid S, Bahat-Dinur A, et al. Necrotizing Otitis Externa-Analysis of 83 Cases: Clinical Findings and Course of Disease. OtolNeurotol . 2019;40(1):56-62. doi:10.1097/MAO.0000000000001986

4. Mahdyoun P, Pulcini C, Gahide I, et al. Necrotizing otitis externa: a systematic review. OtolNeurotol . 2013;34(4):620-629. doi:10.1097/MAO.0b013e3182804aee

5. Hollis S, Evans K. Management of malignant (necrotising) otitis externa. J Laryngol Otol . 2011;125(12):1212-1217. doi:10.1017/S0022215110002550

6. Joshua BZ, Sulkes J, Raveh E, Bishara J, Nageris BI. Predicting outcome of malignant external otitis. OtolNeurotol . 2008;29(3):339-343. doi:10.1097/MAO.0b013e3181661879

7. Loh S, Loh WS. Malignant otitis externa: an Asian perspective on treatment outcomes and prognostic factors. Otolaryngol Head Neck Surg . 2013;148(6):991-996. doi:10.1177/0194599813482107

8. Franco-Vidal V, Blanchet H, Bebear C, Dutronc H, Darrouzet V. Necrotizing external otitis: a report of 46 cases. OtolNeurotol . 2007;28(6):771-773. doi:10.1097/MAO.0b013e31805153bd

9. Cohen D, Friedman P. The diagnostic criteria of malignant external otitis. J Laryngol Otol . 1987;101(3):216-221. doi:10.1017/s0022215100101562

10. American Diabetes Association. Diabetes Care in the Hospital: Standards of Medical Care in Diabetes2019 . Diabetes Care . 2019;42(Suppl 1):S173-S181. doi:10.2337/dc19-S015

11. Peled C, Kraus M, Kaplan D. Diagnosis and treatment of necrotising otitis externa and diabetic foot osteomyelitis - similarities and differences. J Laryngol Otol . 2018;132(9):775-779. doi:10.1017/S002221511800138X

12. Stern Shavit S, Soudry E, Hamzany Y, Nageris B. Malignant external otitis: Factors predicting patient outcomes. Am J Otolaryngol . 2016;37(5):425-430. doi:10.1016/j.amjoto.2016.04.005

13. Lee SK, Lee SA, Seon SW, et al. Analysis of Prognostic Factors in Malignant External Otitis. ClinExpOtorhinolaryngol . 2017;10(3):228-235. doi:10.21053/ceo.2016.00612

14. Carfrae MJ, Kesser BW. Malignant otitis externa. OtolaryngolClin North Am . 2008;41(3):537-ix. doi:10.1016/j.otc.2008.01.004

15. Chen CN, Chen YS, Yeh TH, Hsu CJ, Tseng FY. Outcomes of malignant external otitis: survival vs mortality. ActaOtolaryngol . 2010;130(1):89-94. doi:10.3109/00016480902971247

16. Naghibi M, Smith RP, Baltch AL, et al. The effect of diabetes mellitus on chemotactic and bactericidal activity of human polymorphonuclear leukocytes. Diabetes Res ClinPract . 1987;4(1):27-35. doi:10.1016/s0168-8227(87)80030-x

17. Geerlings SE, Hoepelman AI. Immune dysfunction in patients with diabetes mellitus (DM). FEMS Immunol Med Microbiol . 1999;26(3-4):259-265. doi:10.1111/j.1574-695X.1999.tb01397.x

Table 1- Patients' characteristics.

Table 2- DM related patients' characteristics

Table 3 - DM severity and glycemic control based on need for surgery

Table 4 -DM severity and glycemic control based on duration of hospitalization

Table 5 - Patient's characteristics based on mean blood glucose levels during hospitalization

Figure 1 - Incidence of isolated pathogen based on mean glucose measurements during hospitalization

\section{Hosted file}

Tables.docx available at https://authorea.com/users/343668/articles/470296-diabetes-andglycemic-control-in-necrotizing-otitis-externa-noe

Hosted file 
Table 2.docx available at https://authorea.com/users/343668/articles/470296-diabetes-andglycemic-control-in-necrotizing-otitis-externa-noe

\section{Hosted file}

Table 3.docx available at https://authorea.com/users/343668/articles/470296-diabetes-andglycemic-control-in-necrotizing-otitis-externa-noe

\section{Hosted file}

Table 4.docx available at https://authorea.com/users/343668/articles/470296-diabetes-andglycemic-control-in-necrotizing-otitis-externa-noe

\section{Hosted file}

Table 5.docx available at https://authorea.com/users/343668/articles/470296-diabetes-andglycemic-control-in-necrotizing-otitis-externa-noe

\section{Hosted file}

figure 1.docx available at https://authorea.com/users/343668/articles/470296-diabetes-andglycemic-control-in-necrotizing-otitis-externa-noe 